\title{
ISOMETRIES OF UNBOUNDED FREDHOLM MODULES OVER REDUCED GROUP $C^{*}$-ALGEBRAS
}

\author{
EFTON PARK
}

(Communicated by Palle E. T. Jorgensen)

\begin{abstract}
In an earlier paper, the author defined the isometry group of an unbounded Fredholm module over a unital $C^{*}$-algebra. In this paper, the author studies a class of unbounded Fredholm modules over a reduced group $C^{*}$ algebra, and he shows that the isometry groups of these unbounded Fredholm modules are always compact Lie groups. The author also proves a result about the fixed point algebra of such an isometry.
\end{abstract}

Let $A$ be a unital $C^{*}$-algebra. An unbounded Fredholm module $(\mathscr{H}, D)$ over $A$ consists of

(i) a Hilbert space $\mathscr{H}$ and a representation of $A$ on $\mathscr{H}$;

(ii) an unbounded, self-adjoint operator $D$ on $\mathscr{H}$ such that

(a) the set $\{a \in A:[D, a]$ is densely defined and extends to a bounded operator on $\mathscr{H}\}$ is norm dense in $A$;

(b) $\left(1+D^{2}\right)^{-1}$ is a compact operator.

Unbounded Fredholm modules arise in a variety of contexts, including index theory, $K K$-theory, and cyclic cohomology. In addition, Connes has shown [1] that an unbounded Fredholm module serves as a sort of a "noncommutative" metric for the underlying $C^{*}$-algebra. With this in mind, the author defined in [2] the isometry group of an unbounded Fredholm module:

Definition. Let $A$ be a unital $C^{*}$-algebra and let $(\mathscr{H}, D)$ be an unbounded Fredholm module over $A$. The isometry group of $(\mathscr{H}, D)$ is denoted by Iso $(A, \mathscr{H}, D)$, and is defined to be

$$
\begin{aligned}
& I \operatorname{Iso}(A, \mathscr{H}, D)=\{\phi \in \operatorname{Aut}(A): \text { there exists a unitary } U \text { on } \mathscr{H} \text { such that } \\
& \left.\qquad U D=D U \text { and } \phi(a)=U a U^{*} \text { for every } a \in A\right\} .
\end{aligned}
$$

The justification for calling this group an isometry group comes from the following result (see [2] for details):

Received by the editors October 14, 1993.

1991 Mathematics Subject Classification. Primary 46L85, 46L87, 46L40.

Key words and phrases. Noncommutative topology, noncommutative geometry, automorphisms of $C^{*}$-algebras. 
Theorem. Let $M$ be a compact oriented manifold, equipped with a Riemannian metric $g$, let $L^{2}\left(\Lambda^{*}(M)\right)$ be the Hilbert space of $L^{2}$-forms on $M$, and let $d+d^{*}$ be the de Rham operator on $L^{2}\left(\Lambda^{*}(M)\right)$. Then Iso $\left(C(M), L^{2}\left(\Lambda^{*}(M)\right), d+d^{*}\right)$ is naturally isomorphic to Iso $(M, g)$.

In this paper, we use length functions on a discrete group $\Gamma$ to construct unbounded Fredholm modules over the reduced group $C^{*}$-algebra $C_{r}^{*}(\Gamma)$ and we show that, just as in the case of the isometry group of a compact Riemannian manifold, the isometry groups of these unbounded Fredholm modules are always compact Lie groups. In addition, we show that being an isometry with respect to one of these unbounded Fredholm modules places strong restrictions on what the fixed point algebra can be.

\section{LENGTH FUNCTIONS AND UNBOUNDED FREDHOLM MODULES}

Definition. Let $\Gamma$ be a discrete group. A length function on $\Gamma$ is a map $L: \Gamma \longrightarrow$ $[0, \infty)$ such that

(i) $L(e)=0$, where $e$ is the identity element of $\Gamma$;

(ii) $L\left(g^{-1}\right)=L(g)$ for all $g$ in $\Gamma$;

(iii) $L(g h) \leq L(g)+L(h)$ for all $g, h$ in $\Gamma$.

Word length with respect to a set of generators is obviously an example of a length function.

Given a length function $L$, define $D_{L}$ on $l^{2}(\Gamma)$ by $D_{L}\left(\delta_{g}\right)=L(g) \delta_{g}$, where $\delta_{g}$ denotes the unit point mass at $g \in \Gamma$. Letting $C_{r}^{*}(\Gamma)$ act on $l^{2}(\Gamma)$ in the usual way, it is natural to ask when $\left(l^{2}(\Gamma), D_{L}\right)$ is an unbounded Fredholm module over $C_{r}^{*}(\Gamma)$. The following proposition clarifies Lemma 5 of [1].

Proposition. Let $\Gamma$ be a discrete group and let $L$ be a length function on $\Gamma$. Then $\left(l^{2}(\Gamma), D_{L}\right)$ is an unbounded Fredholm module over $C_{r}^{*}(\Gamma)$ if and only if the range of $L$ is unbounded and discrete and $L$ is finite-to-one.

Proof. Suppose the range of $L$ is unbounded and discrete and $L$ is finite-toone. Clearly $D_{L}$ is an unbounded self-adjoint operator. To show that $\left[D_{L}, X\right]$ extends to a bounded operator on $l^{2}(\Gamma)$ for a dense subset of $C_{r}^{*}(\Gamma)$, it suffices to show that $\left[D_{L}, \lambda_{g}\right]$ extends to a bounded operator. We shall show more; namely, that $\left\|\left[D_{L}, \lambda_{g}\right]\right\|=L(g)$ for all $g$ in $\Gamma$.

Fix $g$ in $\Gamma$. For all $h \in \Gamma$,

$$
\left(\lambda_{g^{-1}} D_{L} \lambda_{g}-D_{L}\right) \delta_{h}=(L(g h)-L(h)) \delta_{h},
$$

and hence

$$
\sup _{h \in \Gamma}|L(g h)-L(h)|=\left\|\lambda_{g^{-1}} D_{L} \lambda_{g}-D_{L}\right\|=\left\|\lambda_{g^{-1}}\left[D_{L}, \lambda_{g}\right]\right\|=\left\|\left[D_{L}, \lambda_{g}\right]\right\| .
$$

To show that $\sup _{h \in \Gamma}|L(g h)-L(h)|=L(g)$, first note that $|L(g e)-L(e)|=$ $L(g)$, so $\sup _{h \in \Gamma}|L(g h)-L(h)| \geq L(g)$. Next, for all $h$ in $\Gamma$,

$$
L(g h)-L(h) \leq L(g)+L(h)-L(h)=L(g) .
$$

On the other hand,

$$
L(h)=L\left(g^{-1} g h\right) \leq L\left(g^{-1}\right)+L(g h)=L(g)+L(g h),
$$


or

$$
-L(g) \leq L(g h)-L(h) .
$$

Thus $\sup _{h \in \Gamma}|L(g h)-L(h)|=L(g)$, as desired.

Finally, we show that $\left(1+D_{L}^{2}\right)^{-1}$ is compact . First note that $\left(1+D_{L}^{2}\right)^{-1} \delta_{g}=$ $\frac{1}{1+L(g)^{2}} \delta_{g}$ for all $g$ in $\Gamma$. It is evident that the conditions imposed on $L$ force $\Gamma$ to be countable; let $g_{1}, g_{2}, \ldots$ be an enumeration of the elements of $\Gamma$. Then $\left(1+D_{L}^{2}\right)^{-1}$ is a diagonal operator with respect to the orthonormal basis $\delta_{g_{1}}, \delta_{g_{2}}, \ldots$, and since $\frac{1}{1+L\left(g_{n}\right)^{2}}$ goes to zero as $n$ goes to infinity, $\left(1+D_{L}^{2}\right)^{-1}$ is compact.

Conversely, suppose that $L$ is a length function on $\Gamma$ such that $\left(l^{2}(\Gamma), D_{L}\right)$ is an unbounded Fredholm module over $C_{r}^{*}(\Gamma)$. Since $\left(1+D_{L}^{2}\right)^{-1}$ is compact, $D_{L}$ has a discrete set of eigenvalues, and hence the range of $L$ is exactly the collection of eigenvalues of $D_{L}$. Therefore the range of $L$ is discrete and unbounded. Furthermore, since each eigenspace of $D_{L}$ is finite dimensional, $L$ is finite-to-one.

\section{MAIN RESUlts}

Throughout this section, $L$ will be a length function on a finitely generated discrete group $\Gamma$ with the property that $\left(l^{2}(\Gamma), D_{L}\right)$ is an unbounded Fredholm module over $C_{r}^{*}(\Gamma)$. In addition, we stipulate the (mild) condition that the identity element $e$ is the only element of length zero.

Lemma. Let $\phi$ be an element of $I s o\left(C_{r}^{*}(\Gamma), l^{2}(\Gamma), D_{L}\right)$. Then there is a unique unitary $U$ on $l^{2}(\Gamma)$ such that $U$ commutes with $D_{L}, U \delta_{e}=\delta_{e}$, and $\phi(X)=$ $U X U^{*}$ for all $X$ in $C_{r}^{*}(\Gamma)$.

Proof. Let $V$ be any unitary on $l^{2}(\Gamma)$ that implements $\phi$ and commutes with $D_{L}$. Since $D_{L}$ is selfadjoint, $V^{*}$ also commutes with $D_{L}$ and hence each spectral subspace of $D_{L}$ is a reducing subspace for $V$. In particular, $\operatorname{ker} D_{L}=$ $\mathbb{C} \delta_{e}$ is reducing for $V$, and hence $V \delta_{e}=\mu \delta_{e}$ for some complex number $\mu$ of modulus 1 . The operator $U=\frac{1}{\mu} V$ clearly satisfies the hypotheses of the lemma. Furthermore, the formula $\phi\left(\lambda_{g}\right) \delta_{e}=U \lambda_{g} U^{*} \delta_{e}=U \delta_{g}$ shows that $U$ is unique.

We shall refer to the unitary described in the lemma as the standard unitary implementing $\phi$.

Theorem 1. Iso $\left(C_{r}^{*}(\Gamma), l^{2}(\Gamma), D_{L}\right)$, endowed with the topology of pointwise convergence, is a compact Lie group.

Proof. Since $\Gamma$ is finitely generated, we can choose a positive real number $R$ with the property that the set $S=\{g \in \Gamma: L(g) \leq R\}$ generates $\Gamma$. Let $\mathscr{V}$ be the span of $\left\{\delta_{g}: g \in S\right\}$; note that $\mathscr{V}$ is finite dimensional. Let $\mathscr{U}(\mathscr{V})$ denote the group of unitary operators on $\mathscr{V}$, and define $F: I s o\left(C_{r}^{*}(\Gamma), l^{2}(\Gamma), D_{L}\right) \longrightarrow$ $\mathscr{U}(\mathscr{V})$ by taking $F(\phi)$ to be the restriction to $\mathscr{V}$ of the standard unitary $U$ implementing $\phi \in I s o\left(C_{r}^{*}(\Gamma), l^{2}(\Gamma), D_{L}\right)$. Since $\mathscr{V}$ is a reducing subspace for $U, F$ is well defined. Moreover, it is easy to check that $F$ is a homomorphism of groups. 
Suppose $F(\phi)=\mathrm{I}$, and let $U$ be the standard unitary implementing $\phi$. Then for each $g$ in $S$,

$$
\phi\left(\lambda_{g}\right) \delta_{e}=U \delta_{g}=F(\phi) \delta_{g}=\delta_{g} .
$$

Since an element of $C_{r}^{*}(\Gamma)$ is uniquely determined by its value on $\delta_{e}, \phi\left(\lambda_{g}\right)=$ $\lambda_{g}$ for all $g$ in $S$, and since $S$ generates $\Gamma, \phi$ must be the identity map on $C_{r}^{*}(\Gamma)$. Therefore $F$ is an injection.

To show that $F$ is continuous, let $\left\{\phi_{\alpha}\right\}_{\alpha \in A}$ be a net in $I s o\left(C_{r}^{*}(\Gamma), l^{2}(\Gamma), D_{L}\right)$ converging to $\phi \in I \operatorname{so}\left(C_{r}^{*}(\Gamma), l^{2}(\Gamma), D_{L}\right)$, and let $g$ be an element of $S$. Then

$\left\|\phi_{\alpha}\left(\lambda_{g}\right)-\phi\left(\lambda_{g}\right)\right\| \geq\left\|\phi_{\alpha}\left(\lambda_{g}\right) \delta_{e}-\phi\left(\lambda_{g}\right) \delta_{e}\right\|=\left\|U_{\alpha} \delta_{g}-U \delta_{g}\right\|=\left\|F\left(\phi_{\alpha}\right) \delta_{g}-F(\phi) \delta_{g}\right\|$. Since the set $\left\{\delta_{g}: g \in S\right\}$ spans $\mathscr{V}$, and since $\mathscr{V}$ is finite dimensional, $F\left(\phi_{\alpha}\right)$ converges to $F(\phi)$ in the norm topology on $\mathscr{U}(\mathscr{V})$.

Next, we show that $F$ is an imbedding. Given $\phi$ in $I s o\left(C_{r}^{*}(\Gamma), l^{2}(\Gamma), D_{L}\right)$ and $g$ in $S, \phi\left(\lambda_{g}\right) \delta_{e}=U \delta_{g}$ is in $\mathscr{V}$, whence

$$
\phi\left(\lambda_{g}\right)=\sum_{h \in S}\left\langle\phi\left(\lambda_{g}\right) \delta_{e}, \delta_{h}\right\rangle \lambda_{h}=\sum_{h \in S}\left\langle F(\phi) \delta_{g}, \delta_{h}\right\rangle \lambda_{h} .
$$

Therefore, if $\left\{\phi_{\alpha}\right\}_{\alpha \in A}$ is a net in $I s o\left(C_{r}^{*}(\Gamma), l^{2}(\Gamma), D_{L}\right)$ and $F\left(\phi_{\alpha}\right)$ converges to $F(\phi)$ for some $\phi$ in $I s o\left(C_{r}^{*}(\Gamma), l^{2}(\Gamma), D_{L}\right)$, then $\left\{\phi_{\alpha}\left(\lambda_{g}\right)\right\}_{\alpha \in A}$ converges to $\phi\left(\lambda_{g}\right)$ for each $g$ in $S$. Since the set $\left\{\lambda_{g}: g \in S\right\}$ generates $C_{r}^{*}(\Gamma)$, and since the maps $\phi_{\alpha}$ and $\phi$ are $C^{*}$-homomorphisms, $\left\{\phi_{\alpha}(X)\right\}_{\alpha \in A}$ converges to $\phi(X)$ for every $X$ in $C_{r}^{*}(\Gamma)$.

We claim that the image of $F$ is closed in $\mathscr{U}(\mathscr{V})$. Suppose $\left\{F\left(\phi_{\alpha}\right)\right\}_{\alpha \in A}$ converges to $V$ in $\mathscr{U}(\mathscr{V})$. Then for all $g$ in $S, \phi_{\alpha}\left(\lambda_{g}\right)=\sum_{h \in S}\left\langle F\left(\phi_{\alpha}\right) \delta_{g}, \delta_{h}\right\rangle \lambda_{h}$ converges to $\sum_{h \in S}\left\langle V \delta_{g}, \delta_{h}\right\rangle \lambda_{h}$, and therefore the net $\left\{\phi_{\alpha}\left(\lambda_{g}\right)\right\}_{\alpha \in A}$ is Cauchy for all $g$ in $\Gamma$. All of the maps $\phi_{\alpha}$ are $C^{*}$-algebra homomorphisms, so $\left\{\phi_{\alpha}(Y)\right\}_{\alpha \in A}$ is Cauchy for all $Y$ in the complex group algebra $\mathbb{C} \Gamma$, and since $\mathbb{C} \Gamma$ is dense in $C_{r}^{*}(\Gamma),\left\{\phi_{\alpha}(X)\right\}_{\alpha \in A}$ is Cauchy for all $X$ in $C_{r}^{*}(\Gamma)$. Define $\phi: C_{r}^{*}(\Gamma) \longrightarrow C_{r}^{*}(\Gamma)$ by $\phi(X)=\lim _{\alpha \in A} \phi_{\alpha}(X)$, and define $U: l^{2}(\Gamma) \longrightarrow l^{2}(\Gamma)$ by $U \delta_{g}=\phi\left(\lambda_{g}\right) \delta_{e}$. For each $\alpha$ in $A$, let $U_{\alpha}$ be the standard unitary implementing $\phi_{\alpha}$. Then $U_{\alpha} \delta_{g}=\phi_{\alpha}\left(\lambda_{g}\right) \delta_{e}$, and therefore $U_{\alpha} \delta_{g}$ converges to $U \delta_{g}$ for each $g$ in $\Gamma$. This implies that for each eigenspace $\mathscr{V}_{l}$ of $D_{L}, U$ restricts to a unitary on $\mathscr{V}_{l}$, and therefore $U$ is a unitary on $l^{2}(\Gamma)$ that commutes with $D_{L}$.

It remains to show that $U$ implements $\phi$. Fix $g$ in $\Gamma$, and let $l=L(g)$. Then $\mathscr{V}_{l}$ is a finite-dimensional reducing subspace for $U$ and each $U_{\alpha}$. By restricting to $\mathscr{V}_{l}$, we see that not only does $U_{\alpha} \delta_{g}$ converge to $U \delta_{g}$ as we noted above, but $U_{\alpha}^{*} \delta_{g}$ converges to $U^{*} \delta_{g}$ as well. Thus $U_{\alpha}$ and $U_{\alpha}^{*}$ converge strongly to $U$ and $U^{*}$, respectively. This in turn implies that $U_{\alpha} \lambda_{h} U_{\alpha}^{*}$ converges strongly to $U \lambda_{h} U^{*}$ for each $h$ in $\Gamma$. But $U_{\alpha} \lambda_{h} U_{\alpha}^{*}=\phi_{\alpha}\left(\lambda_{h}\right)$, so $U \lambda_{h} U^{*}=\phi\left(\lambda_{h}\right)$ for each $h$, as desired.

The homomorphism $F$ is an imbedding of $I s o\left(C_{r}^{*}(\Gamma), l^{2}(\Gamma), D_{L}\right)$ onto a closed subgroup of the compact Lie group $\mathscr{U}(\mathscr{V})$, and therefore Iso $\left(C_{r}^{*}(\Gamma), l^{2}(\Gamma), D_{L}\right)$ is a compact Lie group itself. 
Remark/Example. The assumption that $\Gamma$ is finitely generated cannot be dispensed with. Let $\Gamma=\oplus_{n=1}^{\infty} \mathbb{Z}$ and let $L$ be the length function

$$
L\left(k_{1}, k_{2}, k_{3}, \ldots\right)=\left|k_{1}\right|+2\left|k_{2}\right|+3\left|k_{3}\right|+\cdots \text {. }
$$

Then $\left(l^{2}(\Gamma), D_{L}\right)$ is an unbounded Fredholm module over $C_{r}^{*}(\Gamma)$. For each homomorphism $\chi$ from $\Gamma$ to the circle group $\mathbb{T}$, define an automorphism $\phi_{\chi}$ of $C_{r}^{*}(\Gamma)$ by $\phi_{\chi}\left(\lambda_{g}\right)=\chi(g) \lambda_{g}$. A straightforward computation shows that the unitary $U_{\chi}$ on $l^{2}(\Gamma)$ given by $U_{\chi} \delta_{h}=\chi(g) \delta_{h}$ commutes with $D_{L}$ and implements $\phi_{\chi}$. Thus the group $\operatorname{Hom}(\Gamma, \mathbb{T})$ can be viewed as a subgroup of Iso $\left(C_{r}^{*}(\Gamma), l^{2}(\Gamma), D_{L}\right)$, and since $\operatorname{Hom}(\Gamma, \mathbb{T})$ is clearly infinite-dimensional, Iso $\left(C_{r}^{*}(\Gamma), l^{2}(\Gamma), D_{L}\right)$ is not a Lie group.

Theorem 2. Let $\phi$ be an isometry of $\left(l^{2}(\Gamma), D_{L}\right)$ and let $X$ be a fixed point of $\phi$. Then there exists a sequence $\left\{X_{n}\right\}$ of fixed points in $\mathbb{C} \Gamma$ with the property that $\left\{X_{n} \delta_{e}\right\}$ converges to $X \delta_{e}$ in the norm topology on $l^{2}(\Gamma)$.

Proof. Suppose $\phi(X)=X$, and let $U$ be the standard unitary implementing $\phi$. Then $U X U^{*}=X$, so $U X=X U$, and $U X \delta_{e}=X U \delta_{e}=X \delta_{e}$. On the other hand, suppose that $U X \delta_{e}=X \delta_{e}$. Then the fact that $U^{*} \delta_{e}=\delta_{e}$ gives us $U X U^{*} \delta_{e}=X \delta_{e}$. Since $U X U^{*}$ and $X$ are elements of $C_{r}^{*}(\Gamma)$ that agree on $\delta_{e}, \phi(X)=U X U^{*}=X$. Therefore, $X \in C_{r}^{*}(\Gamma)$ is a fixed point of $\phi$ if and only if $X \delta_{e} \in l^{2}(\Gamma)$ is a fixed point of $U$.

Let $r_{1}, r_{2}, \ldots$ be an enumeration of the eigenvalues of $D_{L}$, and let $P_{k}$ be the orthogonal projection onto the $r_{k}$-eigenspace of $D_{L}$. Since $U$ commutes with $D_{L}, U$ also commutes with $P_{k}$, whence $P_{k} X \delta_{e}$ is a fixed point of $U$ for all $k$. Furthermore, since the range of $P_{k}$ is spanned by the finite set $\left\{\delta_{g}: L(g)=r_{k}\right\}$, it is clear that $P_{k} X$ is a fixed point of $\phi$ that is in $\mathbb{C} \Gamma$. If for each positive integer $n$ we define $X_{n}$ to be $\sum_{k=0}^{n} P_{k} X$, it is easy to see that $\left\{X_{n}\right\}$ is the desired sequence.

Corollary. Let $\phi$ be an isometry of $\left(l^{2}(\Gamma), D_{L}\right)$. If $\phi$ has no nontrivial fixed points in any of the eigenspaces of $D_{L}$, or more generally, has no nontrivial fixed points in $\mathbb{C} \Gamma$, then $\phi$ has no nontrivial fixed points.

\section{REFERENCES}

1. A. Connes, Compact metric spaces, Fredholm modules, and hyperfiniteness, Ergodic Theory Dynamical Systems 9 (1989), 207-220.

2. E. Park, Isometries of noncommutative metric spaces, Proc. Amer. Math. Soc. 123 (1995), 97-105.

Department of Mathematics, Texas Christian University, Fort Worth, Texas 76129

E-mail address: epark@gamma.is.tcu.edu 